\title{
Manifestaciones extrapulmonares de la infección por SARS-CoV-2
}

\author{
Extrapulmonary manifestations of SARS-CoV-2 virus infection
}

\begin{abstract}
Raúl Carrillo-Esper ${ }^{1}$, Rebeca E. Melgar-Bieberach ${ }^{1 *}$, Mauricio Tapia-Salazar ${ }^{1}$, Sarahi A. Jacinto-Flores ${ }^{1}$, Ángela N. Campa-Mendoza ${ }^{1}$, Ángel A. Pérez-Calatayud², Mateo Porres-Aguilar ${ }^{3}$, Jorge R. Carrillo-Córdova ${ }^{4}$, Hazel R. Bracho-Olvera ${ }^{5}$ y Dulce M. Carrillo-Córdova ${ }^{6}$

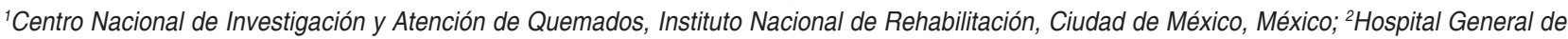
México Dr. Eduardo Liceaga, Ciudad de México, México; ${ }^{3}$ Departamento de Medicina, Universidad de McGill, Division of Adult Thrombosis Medicine and Center of Excellence in Thrombosis and Anticoagulation Care (CETAC), Jewish General Hospital, Quebec, Canadá; ${ }^{4}$ Hospital HMG, Ciudad de México, México; ${ }^{5} \mathrm{Hospital}$ General Dr. Manuel Gea González, Ciudad de México, México; ${ }^{6}$ Instituto Nacional de Ciencias Médicas y Nutrición Salvador Zubirán, Ciudad de México, México
\end{abstract}

\section{Resumen}

La enfermedad causada por un nuevo coronavirus, que inició en 2019, fue denominada COVID-19 y declarada pandemia el 11 de marzo de 2020 por la Organización Mundial de la Salud. Si bien es cierto que los primeros reportes enfatizaron las manifestaciones respiratorias de esta enfermedad como presentación clínica inicial, poco a poco empezaron a aparecer casos con manifestaciones iniciales distintas, involucrando otros sistemas. En los casos en los que se identificó afectación del sistema nervioso central, los hallazgos más frecuentes fueron mareo, cefalea y alteración del estado de alerta. Respecto al sistema cardiovascular, la elevación de biomarcadores cardiacos y la miocarditis son unos de los hallazgos más frecuentes. Los principales síntomas gastrointestinales descritos hasta el momento son anorexia, náuseas, vómitos, diarrea y dolor o disconfort abdominal. La tromboembolia venosa es una complicación frecuente y un problema de salud pública. Las manifestaciones cutáneas siguen siendo un campo de investigación. Se han identificado exantemas maculopapulares, livedo reticular y gangrena acral, entre otros. El personal sanitario debe estar actualizado sobre los nuevos hallazgos clínicos y las formas de presentación de esta enfermedad solo parcialmente conocida, lo que permitirá hacer diagnósticos más precisos y oportunos, y así impactar en el pronóstico de estos enfermos.

Palabras clave: Coagulación intravascular diseminada. Hiposmia. Miocarditis. Neurovirulencia. Tromboembolia venosa.

\begin{abstract}
The disease caused by a new coronavirus, which started in 2019, was named COVID-19 and declared a pandemic on March 11, 2020 by the World Health Organization. Although it is true that the first reports emphasized the respiratory manifestations of this disease as an initial clinical presentation, little by little cases with different initial manifestations began to appear, involving other systems. In cases where central nervous system involvement was identified, the most frequent findings were dizziness, headache, and alteration of alertness. Regarding the cardiovascular system, elevation of cardiac biomarkers and myocarditis are one of the most frequent findings. The main gastrointestinal symptoms described so far are: anorexia, nausea, vomiting,
\end{abstract}

\section{Correspondencia:}

*Rebeca E. Melgar-Bieberach

E-mail: rebecaestela@gmail.com

Calzada México-Xochimilco, 289

Col. Arenal de Guadalupe, Del. Tlalpan

C.P. 14389, Ciudad de México, México
Fecha de recepción: 20-04-2020

Fecha de aceptación: 27-05-2020

DOI: $10.24875 / C I R U .20000363$
Cir Cir. 2020;88(5):654-663

Contents available at PubMed www.cirugiaycirujanos.com
ajo la licencia CC BY-NC-ND 0009-7411/○ 2020 Academia Mexicana de Cirugi
(http://creativecommons.org/licenses/by-nc-nd/4.0). 
diarrhea, abdominal pain and/or discomfort. Venous thromboembolism is a frequent complication and a public health problem. Skin manifestations remain a field of investigation. Maculopapular rashes, reticular livedo, acral gangrene, among others, have been identified. Health personnel must be updated on new clinical findings and the forms of presentation of this partially known disease, which will make it possible to make more accurate and timely diagnoses, thus impacting the prognosis of these patients.

Key words: Disseminated intravascular coagulation. Hyposmia. Myocarditis. Neurovirulence. Venous thromboembolism.

\section{Introducción}

En diciembre de 2019, en la ciudad de Wuhan, provincia de Hubei, en China, se originó una enfermedad que detuvo y acaparó la atención del mundo entero. La enfermedad causada por un nuevo coronavirus, que inició en 2019, fue denominada COVID-19 (COronaVIrus Disease 2019) y declarada pandemia el 11 de marzo de 2020 por la Organización Mundial de la Salud'. El Comité Internacional de Taxonomía de Virus, como órgano que autoriza y organiza la clasificación taxonómica de los virus, asignó el nombre de SARS-CoV-2 (Severe Acute Respiratory Syndrome CoronaVirus 2) a este nuevo virus.

Esta enfermedad se manifestó con síntomas muy similares a los del síndrome respiratorio agudo grave (SARS) identificado por primera vez en Asia en el año 2003; sin embargo, la evidencia reciente ha demostrado que el SARS-CoV-2 puede afectar no solo el sistema respiratorio, sino también otros órganos y sistemas, incluidos el sistema nervioso central (SNC) y periférico (SNP), cardiovascular, gastrointestinal, hepático, renal, hematológico y cutáneo, entre otros.

EI SARS-CoV-2 se ha aislado en secreciones conjuntivales, lágrimas, heces y orina, lo que confirma que la infección no se limita al tracto respiratorio² ${ }^{2}$. Es un virus altamente contagioso y su letalidad varía mucho según la región ${ }^{3}$.

Durante la etapa inicial del brote de COVID-19, el diagnóstico de la enfermedad se complicó por la diversidad de síntomas y la gravedad de la enfermedad en el momento de la presentación. Si bien los síntomas más comunes de la COVID-19 al inicio de la enfermedad incluyen fiebre (88\%), fatiga $(38 \%)$, tos $(67.8 \%)$, disnea $(18.7 \%)$ y mialgia ${ }^{4}$, otros síntomas menos comunes son dolor de cabeza, dolor abdominal, diarrea, náuseas y vómitos.

Esto también se reportó en un metaanálisis realizado por $\mathrm{Ma}$, et al. ${ }^{2}$, en el que se identificaron como síntomas comunes fiebre $(79.1 \%)$, tos $(58.0 \%)$ y fatiga $(29.3 \%)$; los síntomas digestivos, como diarrea (5.7\%), náuseas o vómitos (2.0\%), fueron relativamente raros.
La mayoría de los pacientes infectados sufrirán una forma leve de la enfermedad ${ }^{4}$ y los menores de 15 años generalmente no desarrollarán formas graves y cursarán asintomáticos ${ }^{3}$.

La dificultad de establecer unos síntomas específicos de la infección por SARS-CoV-2 aumenta la posibilidad de llegar a diagnósticos de trabajo erróneos. Esta situación puede estar relacionada con una mayor exposición de personas sanas al virus, incluyendo el personal sanitario, por lo cual se hace imperativa la utilización de las medidas de protección de manera global en el escenario actual de esta pandemia.

Se postula que la realización de intervenciones apropiadas y la instauración del tratamiento de manera oportuna pueden ofrecer un mejor pronóstico.

En la siguiente revisión se abordan, por aparatos y sistemas, las complicaciones extrapulmonares secundarias a la infección por SARS-CoV-2 con la finalidad de presentar al lector un compendio útil en la práctica clínica y aportar a la literatura existente a la fecha.

\section{Manifestaciones neurológicas de la infección por SARS-CoV-2}

EI SARS-CoV-2, desde su origen en la ciudad de Wuhan, China, se extendió rápidamente, dejando dudas sobre su origen, forma de propagación y fisiopatología, además de todas las potenciales manifestaciones clínicas en el ser humano. Se han ido presentando casos que no muestran los síntomas considerados típicos en un inicio, sino que tienen un matiz neurológico, como cefalea, deterioro del estado de alerta, alteraciones de la marcha o datos de focalización central o periférica ${ }^{5}$.

Recientemente se ha identificado al receptor de la enzima convertidora de angiotensina 2 (ECA2) como el receptor funcional del síndrome de distrés respiratorio agudo secundario a la infección por SARSCoV-2. Este receptor se encuentra en numerosos órganos, incluyendo el sistema nervioso (células gliales y neuronas), el hígado, los riñones y el músculo. Por ello, la infección por SARS-CoV-2 puede causar 
síntomas neurológicos por mecanismos directos e indirectos, de modo similar a lo que ocurre con el SARS-CoV y el coronavirus causante del síndrome respiratorio de oriente medio (MERS-CoV). Se ha detectado ácido nucleico del SARS-CoV en el líquido cefalorraquídeo y SARS-CoV-2 en tejido cerebral ${ }^{6}$.

Ambos virus exhiben la proteína en espiga S1, que permite la unión del virión con la membrana celular a través de la interacción con el receptor ECA2 del huésped. La afinidad del SARS-CoV-2 a este receptor es 10 a 20 veces mayor que la del SARS-CoV7. Este antígeno de la superficie viral es el objetivo de los anticuerpos neutralizantes durante la infección ${ }^{8}$.

Dichos receptores también se expresan en los órganos circunventriculares y en las células endoteliales cerebrovasculares, los cuales tienen un papel fundamental en la autorregulación vascular y el flujo sanguíneo cerebral.

Se han descrito diversas posibles rutas de infección al SNC: la propagación a través del bulbo olfatorio, desde los mecanorreceptores y quimiorreceptores en el pulmón y las vías respiratorias bajas, y la vía hematógena a través de los receptores cerebrales de la $\mathrm{ECA}^{9}$.

Es probable que la disfunción de la angiotensina II cerebral esté involucrada en la interrupción de la autorregulación y en los picos de presión arterial que dan como resultado la rotura de la pared arterial ${ }^{10}$.

Hasta el momento no se ha determinado que exista un aumento del riesgo de muerte atribuible al uso crónico de inhibidores de la ECA 2 o de antagonistas del receptor de la angiotensina II.

Las principales vías patogénicas descritas para la afectación del SNC por el SARS-CoV-2 son la patogenicidad viral directa, la patogenicidad inmunomediada dirigida al tejido cerebral, la afectación inflamatoria de los vasos sanguíneos del cerebro y la coagulación intravascular secundaria a la respuesta inflamatoria sistémica como causa principal de trombosis, hemorragia y accidente cerebrovascular ${ }^{9}$.

La diseminación del SARS-CoV-2 en la circulación sistémica o a través de la lámina cribiforme del hueso etmoides hacia la circulación cerebral también puede conducir a afectación del SNC.

Debido a la respuesta inflamatoria que genera la replicación viral en los nervios de la mucosa nasal, uno de los hallazgos tempranos es la alteración en el sentido del olfato o hiposmia. Los estudios indican que el virus se desprende en grandes cantidades de la nariz en pacientes sintomáticos y asintomáticos, de una manera más parecida al virus de la gripe que al virus del SARS ${ }^{3}$. Una vez dentro de los tejidos neuronales se produce daño neuronal y daño al revestimiento endotelial, ocasionando rotura capilar y sangrado ${ }^{7}$.

La anosmia con disgeusia es un hallazgo frecuente en las infecciones respiratorias víricas, como consecuencia de una reacción inflamatoria de la mucosa nasal y la presencia de rinorrea; sin embargo, las observaciones recientes en el actual contexto epidemiológico convierten al SARS-CoV-2 en el principal sospechoso.

En el estudio de Lechien, et al. ${ }^{11}$, el $85.6 \%$ y el $88.0 \%$ de los enfermos informaron disfunción olfativa y gustativa, respectivamente. La mayoría de los pacientes $(65.7 \%)$ reportaron alteraciones del olfato después de la aparición de síntomas generales de oídos, nariz y garganta; sin embargo, el $11.8 \%$ de los pacientes en este estudio informaron que la disfunción olfatoria apareció antes que cualquier otro síntoma, lo que sugiere que la anosmia puede ser importante para la detección temprana de la COVID-1911.

En un esfuerzo por establecer la importancia de estos síntomas en el diagnóstico y la progresión de la enfermedad, la American Academy of Otolaryngology-Head and Neck Surgery ha establecido la Herramienta de Informe de Anosmia COVID-19 para permitir que los médicos de todas las especialidades del mundo ayuden en la detección precoz de casos, reduciendo así la probabilidad de propagación de la enfermedad ${ }^{11}$.

Si bien los efectos de la COVID-2019 en la percepción olfativa y gustativa pueden ser transitorios, se ha planteado la posibilidad de que los virus y otros agentes contaminantes puedan ser la causa iniciadora de enfermedades neurodegenerativas como la enfermedad de Parkinson ${ }^{12}$.

La invasión viral del parénquima pulmonar conduce a trastornos del intercambio gaseoso, causando hipoxia, promoviendo el metabolismo anaerobio y la acumulación de ácido láctico, lo que puede causar vasodilatación de los vasos cerebrales, inflamación celular, edema intersticial y obstrucción del flujo sanguíneo, con la consecuente cefalea secundaria a isquemia o congestión, que puede progresar a hipertensión intracraneal, disfunción cerebral, edema bulbar e incluso coma.

Los investigadores de Huazhong, en Wuhan, China, clasificaron los hallazgos neurológicos en tres clases: síntomas del SNC, síntomas del SNP y síntomas musculoesqueléticos. De los pacientes estudiados, el $36.4 \%$ presentaron síntomas neurológicos. El $24.8 \%$ 
tuvo manifestaciones del SNC, el $8.9 \%$ del SNP y el $10.7 \%$ de músculos esqueléticos. Los hallazgos más frecuentes correspondientes al SNC fueron mareo $(16.8 \%)$, cefalea $(13.1 \%)$ y alteración del estado de alerta, y los secundarios a afección del SNP fueron hipogeusia $(5.6 \%)$ e hiposmia $(5.1 \%)^{13}$.

Más recientemente han surgido otros fenotipos neurológicos que involucran el SNC y el SNP, caracterizados por anosmia, ageusia, encefalitis necrohemorrágica y síndrome de Guillain-Barrét ${ }^{14}$.

La mayoría de las manifestaciones neurológicas ocurrieron de manera temprana en los primeros 2 días de la enfermedad. Los pacientes con más probabilidad de desarrollar manifestaciones neurológicas fueron aquellos con infección grave en comparación con los no graves ( $45.5 \%$ y $30.2 \%$, respectivamente), además de ser pacientes significativamente mayores, con comorbilidad subyacente como hipertensión, diabetes, enfermedad cerebrovascular y cardiaca, cáncer y enfermedad renal crónica ${ }^{13}$.

Otra presentación clínica de compromiso neurológico es la encefalopatía tóxica infecciosa o encefalitis tóxica aguda, que se presenta como un síndrome de disfunción cerebral reversible, con edema cerebral. Los individuos afectados cursarán con desorientación, deterioro del estado de alerta, coma, parálisis y cefalea intensa ${ }^{15}$. Se ha demostrado con anterioridad que las infecciones virales neurotrópicas pueden causar inflamación del parénquima cerebral o provocar encefalitis y respuestas autoinmunitarias dirigidas al cerebro en individuos susceptibles ${ }^{16}$.

El transporte axonal de los virus neurotrópicos puede convertir proteínas intrínsecamente desordenadas en aglutinantes promiscuos, que tendrán la capacidad de formar agregados tóxicos, viajar a lo largo de las vías neuronales y ocasionar la muerte celular en el tejido cerebral ${ }^{16}$.

La atención de pacientes que consultan por síntomas o signos neurológicos debe asegurarse de forma continua principalmente en enfermedades que tienen una morbilidad elevada, como son las cerebrovasculares y las infecciones del SNC.

Los protocolos de actuación se ajustarán en función de la sospecha o la confirmación de infección por SARS-CoV-2. Estos deben aplicarse de forma habitual en los pacientes sin sospecha de infección, y en los casos sospechosos o confirmados de COVID-19 se extremarán las medidas de prevención y se considerará fundamental la intervención de un equipo multidisciplinario (médicos internistas, neumólogos, intensivistas) que permita obtener una valoración adecuada de la situación de salud del paciente y su pronóstico funcional y vital ${ }^{17}$.

\section{Manifestaciones cardiovasculares de la infección por SARS-CoV-2}

EI SARS-CoV-2 puede inducir nuevas patologías cardiacas o exacerbar enfermedades cardiovasculares subyacentes. A esto se suman los efectos cardiovasculares adversos y tóxicos, secundarios a la utilización de medicamentos antipalúdicos y antivirales.

Es importante mencionar que la incidencia reportada de COVID-19 en la literatura, acompañada de comorbilidad subyacente, es de hasta el $26.0 \%$, y la mayoría de los pacientes (65.3\%) tenían enfermedades cardiovasculares y cerebrovasculares².

La infección por SARS-CoV-2 provoca una tormenta de citocinas, inflamación y un estado de hipercoagulación que no solo causan daño al tejido pulmonar ${ }^{18}$, sino que también, en casos graves, provocan la falla de múltiples órganos y la muerte ${ }^{19}$. Ya se ha relacionado el desarrollo de arritmias, lesión miocárdica, inflamación vascular, infarto agudo de miocardio, miocarditis con disfunción sistólica, pericarditis, miopericarditis, derrame pericárdico con o sin taponamiento cardiaco, y tromboembolia pulmonar.

En el estudio realizado por Guo, et al. ${ }^{20}$, de los pacientes con COVID-19 el 27.8\% tenían lesión miocárdica, lo que resultó en disfunción cardiaca y arritmias. Este estudio mostró que la lesión miocárdica tiene una asociación significativa con el desenlace fatal por COVID-19, y que el pronóstico de los pacientes con enfermedad cardiovascular subyacente, pero sin lesión miocárdica, es relativamente favorable ${ }^{20}$; sin embargo, la letalidad en los pacientes con cardiopatía es incierta hasta el momento. En este grupo de pacientes estudiados, la mortalidad fue del $13.33 \%$ para aquellos con enfermedad cardiovascular subyacente y valores normales de troponina $\mathrm{T}$, del $37.50 \%$ para aquellos sin enfermedad cardiovascular subyacente y valores elevados de troponina T, y del $69.44 \%$ para aquellos con enfermedad cardiovascular y valores de troponina $T$ elevados ${ }^{20}$.

En un estudio observacional realizado en Wuhan, China, por Shi, et al. ${ }^{21}$, se identificó lesión cardiaca en el $19.7 \%$ de los pacientes con COVID-19 y fue un predictor independiente de mortalidad hospitalaria $(51,2 \%$ frente a $4,5 \%$ en aquellos sin lesión cardiacá; $p<0.001$ ).

Los estudios de biomarcadores cardiacos sugieren una alta prevalencia de lesión cardiaca en pacientes hospitalizados. La lesión miocárdica probablemente 
esté asociada con miocarditis o isquemia relacionada con la infección, y es un factor pronóstico importan$t^{22}$. Esta puede provocar fibrosis auricular o ventricular, generando así un sustrato para la aparición de arritmias posteriores ${ }^{19}$. La hipoxemia causada por la afección pulmonar por COVID-19 puede desencadenar fibrilación auricular, y esta puede persistir inclusive luego de la mejoría de la función pulmonar.

La retirada brusca de los inhibidores del sistema renina-angiotensina-aldosterona en pacientes de alto riesgo, como por ejemplo aquellos con insuficiencia cardiaca o enfermedad coronaria, puede provocar inestabilidad clínica y resultados adversos para la salud ${ }^{23}$.

Por el momento no hay evidencia clínica ni científica para suspender los tratamientos con inhibidores de la enzima convertidora de angiotensina o con antagonistas del receptor de la angiotensina.

\section{Manifestaciones gastrointestinales de la infección por SARS-CoV-2}

EI SARS-CoV-2 ha mostrado tropismo por el sistema gastrointestinal, particularmente por el intestino. Esto se ha verificado al detectar partículas virales en biopsias y en heces de pacientes, condición que puede explicar en parte los síntomas gastrointestinales y la potencial recurrencia y transmisión de la infección ${ }^{24}$. Los principales síntomas gastrointestinales descritos son anorexia, náuseas, vómitos, diarrea y dolor o disconfort abdominal25.

En Hong Kong, según Shing Cheung, et al. ${ }^{25}$, de 59 pacientes estudiados con diagnóstico de COVID-19, el $25.4 \%$ presentó síntomas gastrointestinales y en el $15.3 \%$ se aisló RNA del virus en las heces. Se detectó RNA viral con mayor frecuencia en enfermos con diarrea que en aquellos que no cursaron con esta manifestación clínica.

En contraste con lo publicado por shing Cheung, et al. ${ }^{25}$, Sultan, et al. ${ }^{26}$ reportan que los síntomas gastrointestinales están asociados a COVID-19 en menos del $10 \%$ de los casos. Este porcentaje se obtuvo al realizar un análisis de la prevalencia de diarrea, náuseas, vómitos, dolor abdominal y alteraciones de las pruebas de función hepática, a través de un metaanálisis de 47 estudios que incluyó 10,890 pacientes, basándose en las recomendaciones de buena práctica clínica para la gestión participativa de la COVID-19.

En el estudio desarrollado en Hubei, China, por Pan et al. ${ }^{27}$, sobre las características clínicas de pacientes con COVID-19 y síntomas digestivos, se reportó que los pacientes con síntomas digestivos tenían valores medios de enzimas hepáticas más elevados, recuentos de monocitos más bajos y mayor tiempo de protrombina, y requirieron más tratamiento antibiótico que los pacientes sin esta sintomatología.

La presencia de ácidos nucleicos del SARS-CoV-2 en tejido hepático ha confirmado la infección directa. EI SARS-CoV-2 comparte un $82 \%$ de similitud con el genoma del SARS-CoV, y se ha descrito una incidencia de compromiso hepático de hasta el $60 \%$ en los pacientes infectados con este último. En los pacientes con COVID-19 grave se ha observado mayor incidencia de afección hepática ${ }^{28}$.

En el estudio llevado a cabo por Guan, et al. ${ }^{4}$ se observó que el $18.2 \%$ de los pacientes con enfermedad no grave y el $39.4 \%$ de aquellos con enfermedad grave tenían elevación de la aspartato aminotransferasa. Hay mayor incidencia de elevación de la aspartato aminotransferasa en el grupo de pacientes con enfermedad grave. La alanino aminotransferasa también mostró una mayor incidencia de aumento en el grupo con enfermedad grave (28.1\%) en comparación con el grupo con enfermedad no grave (19.8\%). Hasta el $60 \%$ de los pacientes infectados tendrán lesión hepática leve o moderada, caracterizada por elevación de las transaminasas, hipoproteinemia y prolongación del tiempo de protrombina ${ }^{24}$.

Según la British Society of Gastroenterology ${ }^{29}$, hay procedimientos que deberán continuar realizándose en los pacientes con COVID-19, como es el caso de las intervenciones en el sangrado digestivo alto, la presencia de cuerpos extraños en el tracto digestivo superior, las lesiones obstructivas del tracto digestivo inferior, la obstrucción biliar aguda y el sangrado digestivo bajo en caso de que no se pueda resolver por radiología intervencionista.

La relevancia de la aparición de síntomas gastrointestinales como primera manifestación de la enfermedad es la prolongación del tiempo previo a la consulta e inclusive la admisión al hospital, como se demostró en el estudio realizado por Pan, et al. ${ }^{27}$. Se observó que los pacientes con síntomas digestivos tuvieron un tiempo significativamente más largo desde el inicio de los síntomas hasta su ingreso al hospital, en comparación con aquellos sin síntomas digestivos (9 vs. 7,3 días). Este hallazgo puede estar asociado a un inicio tardío del tratamiento y a peores resultados clínicos.

Se requiere la realización de estudios clínicos que incluyan la aplicación de cuestionarios estandarizados y dirigidos a la evaluación de los síntomas 
gastrointestinales, así como la medición de las pruebas de función hepática al momento de la admisión hospitalaria, para poder establecer mejor su incidencia y su asociación a la COVID-1926.

\section{Afectación renal por la infección por SARS-CoV-2}

La COVID-19 puede afectar múltiples órganos, entre ellos el riñón ${ }^{30}$. En informes anteriores de infecciones por SARS y MERS-CoV, la lesión renal aguda (LRA) se desarrolló en el 5-15\% de los casos y tuvo una alta tasa de mortalidad (60-90\%). Los primeros informes sugirieron una menor incidencia (3-9\%) de LRA en los pacientes con COVID-194,31,32. La etiología de la afectación de la función renal en la COVID-19 es multifactorial.

EI SARS-CoV-2 puede ejercer efectos citopáticos directos sobre el tejido renal. Esto está respaldado por la detección de fragmentos de coronavirus en sangre y orina, tanto en los pacientes con el virus del SARS de $2003^{33}$ como en aquellos con SARS-CoV- $2^{34}$. La lesión renal también puede ser causada por la entrada del virus a las células renales.

Datos recientes de secuenciación de ARN de tejido humano demostraron que la ECA2 se expresa en el riñón casi 100 veces más que en el pulmón ${ }^{35}$. Los mecanismos de afectación renal se pueden clasificar en tres grupos que se encuentran interconectados:

- Daño por citocinas: el síndrome de liberación de citocinas, denominando también "tormenta de citocinas", ocurre en la sepsis, el síndrome hemofagocítico y la terapia con células $T$ del receptor de antígeno quimérico ${ }^{36}$. En pacientes con síndrome de liberación de citocinas, la LRA puede ocurrir como resultado de la inflamación intrarrenal, el aumento de la permeabilidad vascular, la disminución del volumen y la cardiomiopatía, lo que puede conducir al síndrome cardiorrenal de tipo 1. Este síndrome incluye lesión endotelial sistémica, que se manifiesta clínicamente como derrames pleurales, edema, hipertensión abdominal, pérdida de líquido a un tercer espacio, depleción de líquido intravascular e hipotensión. La interleucina 6 proinflamatoria se considera la citocina más importante en el síndrome de liberación de citocinas. En los pacientes con síndrome de distrés respiratorio agudo por SARS-CoV-2, la concentración plasmática de interleucina 6 aumenta de manera significativa ${ }^{37}$. Las terapias extracorpóreas también se han propuesto para eliminar las citocinas en pacientes con sepsis y podrían ser beneficiosas en pacientes graves con COVID-1938, al poder prevenir el daño a los órganos inducido por el síndrome de liberación de citocinas.

- Disfunción de órganos: según el estudio de Ronco y Rei ${ }^{39}$, la edad avanzada, la gravedad de la enfermedad, la diabetes mellitus y el balance positivo de líquidos se asociaron de manera independiente al desarrollo de LRA. La gravedad de la LRA se asoció a la edad avanzada, la diabetes mellitus, el índice de masa corporal elevado, los antecedentes de insuficiencia cardiaca, la mayor presión máxima en las vías respiratorias y el puntaje de SOFA (Sequential Organ Failure Assessment Score) más elevado.

- Efectos sistémicos: la administración de fluidos puede conducir a un balance hídrico positivo en pacientes en estado de shock, empeorando la congestión de la vena renal, lo que lleva al síndrome del compartimento renal. Los pacientes con COVID-19 grave desarrollan shock séptico. La LRA asociada a sepsis puede actuar sinérgicamente con otros mecanismos de daño renal ${ }^{4}$.

En pacientes con infecciones bacterianas gramnegativas sospechadas o confirmadas, el uso de hemoperfusión con un cartucho que contiene fibras de poliestireno con polimixina B proporciona una adsorción de endotoxina efectiva ${ }^{40}$, estrategia que ya está siendo utilizada en pacientes con infección por el SARS-CoV-2.

En un estudio realizado en Wuhan, China, se observó que más del $40 \%$ de los pacientes tenía evidencia de enfermedad renal, con valores elevados de creatinina sérica y de nitrógeno ureico en sangre en más del $13 \%$. La presencia de enfermedad renal se asoció con una mayor mortalidad hospitalaria ${ }^{41}$.

Los estudios a la fecha muestran que la prevalencia de enfermedad renal al ingreso y el desarrollo de LRA durante la hospitalización en pacientes con COVID-19 es alta. La LRA es una de las principales causas de gravedad de la enfermedad, contribuyendo al fallo multiorgánico y la muerte. La monitorización de la función renal es de suma importancia, como lo es también el inicio de tratamiento continuo y precoz del fallo renal con terapia de reemplazo renal continuo.

Entre las características basales de los pacientes infectados con SARS-CoV-2, la gravedad de la enfermedad y la mortalidad $(20.2 \%$ y $3.1 \%$ ) están altamente asociadas a otras enfermedades subyacentes, como hipertensión, diabetes, enfermedades cerebrovasculares, 
enfermedad pulmonar obstructiva crónica y enfermedad renal crónica, en comparación con los casos no graves. Esto sugiere que los pacientes con tales padecimientos son especialmente vulnerables a la infección, por lo que deben recibir más atención y cuidado².

\section{Alteraciones de la coagulación producidas por la infección por SARS-CoV-2}

Existen ya ciertos aspectos a considerar respecto a las alteraciones de la coagulación que son de gran importancia. La enfermedad grave comúnmente está asociada a coagulopatía ${ }^{42}$.

La coagulopatía en la sepsis grave y en el choque séptico en el contexto clínico de la COVID-19 grave representa una complicación relativamente frecuente, caracterizada en especial por una elevación significativa de los valores de dímero D. El 50\% de los pacientes con COVID-19 presentan valores elevados de dímero $D$ durante la progresión de la enfermedad, y esta proporción puede llegar a ser tan alta como del $100 \%$ en los casos de muerte. El nivel de dímero $D$ en los pacientes graves es significativamente más alto que en los pacientes con enfermedad leve. Algunos pacientes se deterioran repentinamente durante el tratamiento, lo que sugiere que aquellos con enfermedad grave podrían tener un mayor riesgo de trombosis ${ }^{4,42}$.

Los cambios patológicos descritos en los pacientes con COVID-19 se concentran principalmente en los pulmones, donde los vasos sanguíneos alveolares septales se encuentran congestionados y con edema, y se puede ver una clara formación de trombos. Estos trombos de fibrina son diferentes de los trombos blancos en los pulmones de los pacientes con SARS ${ }^{43}$.

El síndrome de liberación de citocinas desencadenado por el virus puede estar relacionado con el síndrome de activación del macrófago o con un síndrome antifosfolípido, como parte de los síndromes hiperferritinémicos. La presencia de daño microvascular difuso, con el consecuente fallo orgánico múltiple, es una causa importante de muerte en los pacientes graves con COVID-1931-34,42.

En un análisis retrospectivo realizado por Tang, et al. ${ }^{42}$ se compararon los parámetros de coagulación de sobrevivientes y no sobrevivientes. El tiempo promedio desde la admisión hasta la identificación de coagulación intravascular diseminada (CID) fue de 4 días. Al momento del ingreso, los no sobrevivientes mostraron niveles significativamente mayores de dímero $\mathrm{D}$ y un tiempo de protrombina (TP) más prolongado, en comparación con los sobrevivientes. En este mismo estudio se asoció el uso de heparina de bajo peso molecular con un mejor pronóstico.

A diferencia del patrón clásico observado en la sepsis, a menudo la elevación del tiempo parcial de tromboplastina activada es menor que la elevación del TP, probablemente debido al aumento de los niveles del factor VIII. La trombocitopenia es leve y no se identifica microangiopatía. La incidencia de trombocitopenia en pacientes con COVID-19 es variable. Se ha descrito que algunos pacientes con enfermedad grave desarrollaron coagulopatía con activación fulminante de la coagulación y consumo de factores.

\section{Complicaciones trombóticas y tromboembolia venosa en la COVID-19 grave}

La activación de la cascada de coagulación, tanto sistémica como de la vasculatura arterial pulmonar, representa un mecanismo fisiopatogénico esencial del huésped ante la sepsis.

Es importante enfatizar la interacción que existe entre los diferentes componentes celulares sanguíneos, el endotelio vascular y el sistema inmunitario, con el fin de promover la síntesis de trombina, que a su vez active y expanda dicha cascada de la coagulación. Este fenómeno complejo se conoce actualmente con el nombre de «inmunotrombosis» 44,45 .

Tang, et al. ${ }^{42}$ observaron, en 183 pacientes con COVID-19 grave, que la presencia de coagulopatía inducida por sepsis (CIS) y de CID graves, definidas por los criterios actualizados y recientemente validados por la International Society on Thrombosis and Haemostasis con un puntaje $>4$ puntos en la COVID-19 grave, se asocian a un peor pronóstico y un aumento de la mortalidad a corto plazo; el $71.4 \%$ de los fallecidos mostraron evidencia de haber desarrollado CIS o CID, frente al $0.6 \%$ de los sobrevivientes ${ }^{42,46}$.

Guan, et al. $^{4}$ observaron valores de dímero $D \geq 500 \mathrm{ng} / \mathrm{ml}$ en el $43 \%$ de los pacientes no graves, en comparación con el $60 \%$ de los pacientes con enfermedad grave.

Un metaanálisis de nueve estudios que incluyó 400 pacientes con COVID-19 grave demostró la asociación de trombocitopenia con enfermedad muy grave, así como un incremento de hasta cinco veces en el riesgo de desarrollar COVID-19 muy grave con admisión directa a una unidad de cuidados intensivos; sin embargo, los autores concluyen que la trombocitopenia no fue un parámetro consistente al inicio de la 
presentación hospitalaria que estuviera asociado a mortalidad significativa ${ }^{47}$.

La tromboembolia venosa (TEV), ya sea trombosis venosa profunda o tromboembolia pulmonar aguda, es una complicación frecuente y un problema de salud pública de gran relevancia clínica. Los pacientes con enfermedad neumónica grave por COVID-19 con CIS en estado crítico representan un subgrupo muy vulnerable, a quienes la inflamación del endotelio vascular, sistémico y pulmonar, les confiere un riesgo elevado significativo para el desarrollo de TEV.

En los pacientes con hipoxemia refractaria y deterioro hemodinámico significativo se presenta la necesidad de pensar en TEV, en particular en aquellos con factores de riesgo como elevación de la proteína $C$ reactiva, la ferritina y el dímero D, un TP prolongado, trombocitopenia, productos de degradación de la fibrina, interleucina 6, etc., con alta sospecha clínica de CIS o CID en el contexto de un síndrome de distrés respiratorio ${ }^{19}$.

Cui, et al. ${ }^{48}$ han reportado una incidencia de TEV del $25 \%$. Estos investigadores observaron que los valores de dímero $D>1500 \mathrm{ng} / \mathrm{ml}$ representaban un buen índice pronóstico para la identificación de TEV de alto riesgo. Sin embargo, cabe destacar que en este estudio ningún paciente recibió inicialmente tromboprofilaxis.

Klok, et al. ${ }^{49}$ identificaron una incidencia total acumulada del $31 \%$ de eventos trombóticos analizando pacientes con COVID-19 grave, con neumonía, admitidos a la unidad de cuidados intensivos. Todos los pacientes recibieron tromboprofilaxis apropiada. La tromboembolia pulmonar aguda fue la complicación de TEV más frecuente $(n=25 ; 81 \%)$, y los factores de riesgo importantes para desarrollar TEV fueron la edad y la evidencia objetiva de coagulopatía, caracterizada por una elevación del TP > 3 segundos por arriba de los controles y alteraciones del tiempo parcial de tromboplastina $>5$ segundos por arriba de lo normal.

\section{Manifestaciones cutáneas de la COVID-19}

Ya se han documentado casos con manifestaciones dermatológicas, principalmente en Italia y China. Debido a la alta tasa de contagio, la fotografía clínica se limita y la introducción del equipo necesario para realizarla no es prioritario; por ende, el material visual es escaso.

En Lombardía, Italia, se realizaron esfuerzos para documentar si los pacientes hospitalizados por COVID-19 tenían manifestaciones cutáneas ${ }^{50}$. Se analizaron 148 pacientes con COVID-19, de los cuales
60 se excluyeron por consumo reciente de nuevos medicamentos, ya que la ingesta de fármacos es una de las causas más comunes de eflorescencias y dermatosis en los pacientes hospitalizados. El 20.4\% desarrollaron manifestaciones cutáneas. La región afectada con mayor frecuencia fue el tronco. No se encontró ninguna relación entre las manifestaciones cutáneas y la gravedad de la enfermedad ${ }^{50}$.

No hay reportes de exantemas maculopapulares para otros coronavirus, como el SARS-COV y el MERS-COV 51 .

En Tailandia, se ha reportado el caso de un paciente que se presentó con un sarpullido con petequias ${ }^{49}$. En el contexto epidemiológico de aquel país, el diagnóstico más probable era dengue, pero el paciente desarrolló síntomas respiratorios y finalmente se hizo el diagnóstico de COVID-19.

En España, una paciente de 84 años que se presentó con neumonía por SARS-CoV-2 fue hospitalizada. A su ingreso recibiótratamiento con hidroxicloroquina y lopinavir/ritonavir, y 3 días después desarrolló una dermatosis diseminada a las regiones flexurales, caracterizada por máculas milimétricas eritematosas violáceas con coalescencia en ciertos puntos. No se puede aseverar que la causa haya sido la COVID-19, pero es altamente probable, ya que los mismos fármacos los han tomado miles de pacientes y no se han reportado manifestaciones cutáneas ${ }^{52}$.

Los exantemas virales inespecíficos son los más comunes, tanto en niños como en adultos. Las principales causas en la infancia son adenovirus, virus parainfluenza y virus sincitial respiratorio ${ }^{53}$; en los adultos, la causa más frecuente de exantemas maculopapulares son los fármacos, aunque muchas veces es imposible hacer una distinción entre estos dos grupos usando únicamente la clínica, pues incluso en el estudio histopatológico son muy parecidos ${ }^{51}$. En muchas ocasiones no se encuentra la causa (exantemas idiopáticos $)^{54}$ y la mayoría se resuelven en un periodo de 7 días $^{53}$. La historia clínica y las pruebas virales pueden ayudar a distinguir otras posibles causas, pero dada la situación actual se dificulta la realización de estas intervenciones no cruciales.

Otros reportes hicieron hincapié en los pacientes de cuidados críticos en Wuhan, China, y se informó de acrocianosis parda y gangrena seca ${ }^{55}$.

En Italia se han documentado casos de pacientes pediátricos y adolescentes con lesiones acrales isquémicas $^{56}$. Estas lesiones se caracterizan por afectar los dedos de los pies y a veces las plantas. Comienzan como manchas violáceas que evolucionan a ampollas 
de contenido seroso y luego úlceras de bordes necróticos que afortunadamente se restituyeron en todos los pacientes. Las lesiones son dolorosas y tienen una duración aproximada de 2 semanas. Afectan principalmente a niños al final de la primera década de la vida y a adolescentes.

En muchos casos no se pudo hacer la prueba confirmatoria con reacción en cadena de la polimerasa dada la naturaleza de la emergencia sanitaria, ni tampoco estudios de laboratorios o biopsias de piel. Sin embargo, estos reportes concuerdan temporalmente con la epidemia.

Los diagnósticos diferenciales incluyen acrocianosis por causas cardiorrespiratorias, perniosis lúpica y vasculitis tipo Henoch-Schönlein, pero la morfología es distinta.

También es necesario tomar en cuenta la CID, la deficiencia de proteína $S$ y la meningococemia; sin embargo, estas afecciones se caracterizan por el estado grave del paciente y las lesiones son mucho más extensas.

Otro tipo de lesiones reportadas que traducen una afectación vascular son las de la livedo reticularis transitoria. La livedo reticularis está ocasionado por la reducción del flujo sanguíneo microvascular, lo que condiciona la acumulación de sangre desoxigenada en el plexo venoso. En los casos asociados a COVID-19 que reportan Manalo, et al..$^{55}$ es transitoria. Estos autores postulan que todas las manifestaciones de hipercoagulabilidad asociadas a la infección por SARS-COV-2 se deben a microtrombosis que afectan distintos órganos, incluyendo la piel. No se realizaron biopsias en los pacientes reportados debido a la naturaleza transitoria del fenómeno ${ }^{56}$.

El personal a cargo de los pacientes con esta afección debe estar alerta sobre las presentaciones cutáneas con el objetivo de identificarlas de forma temprana y realizar las intervenciones pertinentes.

\section{Conclusión}

La presentación de síntomas en los enfermos afectados por esta enfermedad no sigue ningún tipo de patrón hasta el momento, y las manifestaciones clínicas comprenden una amplia variedad de aparatos y sistemas, desde pacientes asintomáticos, pasando por pacientes con síntomas extrapulmonares hasta pacientes con sintomatología casi exclusivamente respiratoria.

Esta revisión presenta al lector un compendio de las manifestaciones extrapulmonares de la infección por SARS-CoV-2, y señala la importancia de buscar activamente la presencia de síntomas y signos clínicos no respiratorios en los pacientes que consultan, en el escenario al que nos enfrentamos en todo el mundo, con el objetivo de mejorar el abordaje diagnóstico y terapéutico.

\section{Financiamiento}

Los autores no recibieron patrocinio para llevar a cabo este artículo. No contaron con financiamientos, becas ni soportes económicos para la realización del presente trabajo.

\section{Conflicto de intereses}

Los autores declaran que no existe ningún conflicto de intereses.

\section{Bibliografía}

1. Organización Mundial de la Salud. Alocución de apertura del Director General de la OMS. 2020. (Consultado el 13 de abril de 2020.) Disponible en: https://www.who.int/es/dg/speeches/detail/who-director-general-sopening-remarks-at-the-media-briefing-on-covid-19---11-march-2020.

2. Ma C, Gu J, Hou P, Zhang L, Bai Y, Guo Z, et al. Incidence, clinical characteristics and prognostic factor of patients with COVID-19: a systematic review and meta-analysis. medRxiv; 20 de marzo de 2020. Disponible en: https://www.medrxiv.org/content/10.1101/2020.03.17.20037572v1.

3. De Soto J, Hakim S, Boyd F. The pathophysiology of virulence of the COVID-19 virus. Preprints; 2020. Disponible en: https://www.preprints. org/manuscript/202004.0077/v1.

4. Guan W, Ni Z, Hu Y, Liang W, Ou C, He J, et al. Clinical characteristics of 2019 novel coronavirus infection in China. $N$ Engl $J$ Med. 2020;382:1708-20.

5. Wang HY, Li XL, Yan ZR, Sun XP, Han J, Zhang BW. Potential neurological symptoms of COVID-19. Ther Adv Neurol Disord. 2020;13:1-2.

6. Jin H, Hong C, Chen S, Zhou Y, Wang Y, Mao L, et al. Consensus for prevention and management of coronavirus disease 2019 (COVID-19) for neurologists. Stroke Vasc Neurol. 2020 Apr 1:svn-2020-000382.

7. Mannan Baig A, Khaleeq A, Ali U, Syeda H. Evidence of the COVID-19 virus targeting the CNS: tissue distribution, host-virus interaction, and proposed neurotropic mechanisms. ACS Chem Neurosci. 2020;11:995-8.

8. Bosch BJ, Zee Rvd, Haan CAMd, Rottier PJM. The coronavirus spike protein is a class i virus fusion protein: structural and functional characterization of the fusion core complex. J Virol. 2003;77:8801-11.

9. Natoli S, Oliveira V, Calabresi P, Maia LF, Pisani A. Does SARS $₫$ Cov $\square 2$ invade the brain? Translational lessons from animal models. Eur J Neurol. 2020 Apr 25. doi: 10.1111/ene.14277. Online ahead of print.

10. Sharifi-Razavi A, Karimi N, Rouhani N. COVID 19 and intra cerebral hemorrhage: causative or coincidental. New Microbes New Infect. 2020;35:100669.

11. Lechien R, Chiesa- Estomba CM, De Siati DR, Horoi M, Le Bon S, Rodriguez $\mathrm{A}$, et al. Olfactory and gustatory dysfunctions as a clinical presentation of mild-to-moderate forms of the coronavirus disease (COVID-19): a multicenter European study. Eur Arch Otorhinolaryngol. 2020 Apr 6:1-11. doi: 10.1007/s00405-020-05965-1. Online ahead of print.

12. Jang H, Boltz DA, Webster RG, Smeyne RJ. Viral Parkinsonism. Biochim Biophys Acta. 2009;1792:714-21.

13. Mao L, Wang M, Chen S, He Q, Chang J, Hong C, et al. Neurological manifestations of hospitalized patients with COVID-19 in Wuhan, China. A retrospective case series study. JAMA Neurol. 2020 Apr 10;e201127. doi: 10.1001/jamaneurol.2020.1127. Online ahead of print.

14. Zhao H, Shen D, Zhou H, Liu J, Chen S. Guillain-Barré syndrome associated with SARS-CoV-2 infection: causality or coincidence? Lancet Neurol. 2020;19:383-4.

15. Wu Y, Xu X, Chen Z, Duan J, Hashimoto K, Yang L, et al. Nervous system involvement after infection with COVID-19 and other coronaviruses. Brain Behav Immun. 2020 Mar 30:S0889-1591(20)30357-3. doi: 10.1016/j.bbi.2020.03.031. Online ahead of print. 
16. Savarin C, Bergmann CC. Viral-induced suppression of self-reactive T cells: lessons from neurotropic coronavirus-induced demyelination. J Neuroimmunol. 2017;308:12-6.

17. Muñiz Castrillo S, Porta Etessam J. Asistencia neurológica durante la pandemia de COVID-19. En: Ezpeleta D, Garcia Azorin D, editores. Manual COVID-19 para el neurólogo general. Madrid: Sociedad Española de Neurología; 2020. p. 73-6.

18. Nunes Kochi A, Tagliari AP, Battista Forleo G, Fassini GMTC. Cardiac and arrhythmic complications in patients with COVID-19. J Cardiovasc Electrophysiol. 2020;31:1003-8.

19. Driggin E, Madhavan MV, Bikdeli B, Chuich T, Laracy J, Bondi-Zoccai G, et al. Cardiovascular considerations for patients, health care workers, and health systems during the coronavirus disease 2019 (COVID-19) pandemic. J Am Coll Cardiol. 2020;75:2352-71.

20. Guo T, Fan Y, Chen M, Wu X, Zhang L, He T, et al. Cardiovascular implications of fatal outcomes of patients with coronavirus disease 2019 (COVID-19). JAMA Cardiol. 2020 Mar 27:e201017. doi: 10.1001/jamacardio.2020.1017. Online ahead of print.

21. Shi S, Qin M, Shen B, Cai Y, Liu T, Yang F, et al. Association of cardiac injury with mortality in hospitalized patients with COVID-19 in Wuhan, China. JAMA Cardiology. 2020 Mar 25:e200950. doi: 10.1001/jamacardio.2020.0950. Online ahead of print.

22. Madjid M, Safavi-Naeini $P$, Solomon S, Vardeny $P$. Potential effects of coronaviruses on the cardiovascular system. A review. JAMA Cardiol. 2020 Mar 27. doi: 10.1001/jamacardio.2020.1286. Online ahead of print.

23. Vaduganathan M, Vardeny $O$, Michel T, McMurray JJV, Pfeffer MA Solomon S. Renin-angiotensin-aldosterone system inhibitors in patients with Covid-19. N Engl J Med. 2020;382:1653-9.

24. Gu J, Han B, Wang J. COVID-19: gastrointestinal manifestations and potential fecal-oral transmission. Gastroenterology. 2020;158:1518-9.

25. Shing Cheung K, Hung I, Chan P, Lung KC, Tso E, Liu R, et al. Gastrointestinal manifestations of SARS-CoV-2 infection and virus load in fecal samples from the Hong Kong cohort and systematic review and meta-analysis. Gastroenterology. 2020; $2020 \mathrm{Apr}$ 3;S0016-5085(20)30448-0. doi: 10.1053/j.gastro.2020.03.065. Online ahead of print.

26. Sultan S, Altayar O, Siddique SM, Davitkov P, Feuerstein JD, Lim JK et al. AGA Institute rapid review of the GI and liver manifestations of COVID-19, meta-analysis of international data, and recommendations for the consultative management of patients with COVID-19. Gastroenterology. 2020 May 5;S0016-5085(20)30593-X. doi: 10.1053/j.gastro.2020.05.001. Online ahead of print.

27. Pan L, Mu M, Yang P, Sun Y, Wang R, Yan J, et al. Clinical characteristics of COVID-19 patients with digestive symptoms in Hubei, China: a descriptive, cross-sectional, multicenter study. Am J Gastroenterol. 2020;115:766-73.

28. Zhang S, Shi L, Wang FS. Liver injury in COVID-19: management and challenges. Lancet Gastroenterol Hepatol. 2020;5:428-30.

29. Wang D, Hu B, Hu C, Zhu F, Liu X, Zhang J, et al. Clinical characteristics of 138 hospitalized patients with 2019 novel coronavirus-infected pneumonia in Wuhan, China. JAMA. 2020;323:1061-9.

30. British Society of Gastroenterology (BSG). Endoscopy activity and COVID-19: Bsg and JAG guidance. Consultado Abril 2020. Disponible en: https://www.bsg.org.uk/covid-19-advice/endoscopy-activity-and-covid-19-bsg-and-jag-guidance/

31. Chen N, Zhou M, Dong X, Qu J, Gong F, Han Y, et al. Epidemiological and clinical characteristics of 99 cases of 2019 novel coronavirus pneumonia in Wuhan, China: a descriptive study. Lancet. 2020;395:507-13.

32. Cheng Y, Luo R, Wang K, Zhang M, Wang Z, Dong L, et al. Kidney disease is associated with in hospital death of patients with COVID-19. Kidney Int. 2020;97:829-38.

33. Peiris JS, Chu CM, Cheng VC, Chan KS, Hung IF, Poon LL, et al. Clinical progression and viral load in a community outbreak of coronavirus-associated SARS pneumonia: a prospective study. Lancet. 2003;361:1767-72.

34. Huang C, Wang Y, Li X, Ren L, Zhao J, Hu Y, et al. Clinical features of patients infected with 2019 novel coronavirus in Wuhan, China. Lancet. 2020;395:497-506.
35. Anti-2019-nCoV Volunteers, Li Z, Wu M, Yao J, Guo J, Liao X, Song S, et al. Caution on kidney dysfunctions of 2019-nCoV patients. medRxiv; 2020. Disponible en: https://www.medrxiv.org/content/10.1101/2020.02. $08.20021212 \mathrm{v} 2$.

36. Neelapu S, Tummala S, Kebriaei P, Wierda W, Gutiérrez C, Locke F et al. Chimeric antigen receptor $T$ cell therapy - assessment and management of toxicities. Nature Rev Clin Oncol. 2018;15:47-62.

37. Wu C, Chen X, Cai Y, Xia J, Zhou X, Xu S, et al. Risk factors associated with acute respiratory distress syndrome and death in patients with coronavirus disease 2019 pneumonia in Wuhan, China. JAMA Intern Med. 2020 Mar 13;e200994. doi: 10.1001/jamainternmed.2020.0994. Online ahead of print.

38. Ronco C, Reis T, De Rosa S. Coronavirus epidemic and extracorporeal therapies in intensive care: si vis pacem para bellum. Blood Purific. 2020;49:255-8.

39. Ronco C, Rei T. Kidney involvement in COVID-19 and rationale for extracorporeal therapies. Nat Rev Nephrol. 2020;16:308-10.

40. Klein D, Foster D, Walker P, Bagshaw S, Mekonnen H, Antonelli M. Polymyxin $B$ hemoperfusion in endotoxemic septic shock patients without extreme endotoxemia: a post hoc analysis of the EUPHRATES trial. Intensive Care Med. 2018;44:2205-12.

41. Cheng Y, Luo R, Wang K, Zhang M, Wang Z, Dong L, et al. Kidney impairment is associated with in-hospital death of COVID-19 patients. MedRxiv; 2020. Disponible en: https://www.medrxiv.org/content/10.1101 /2020.02.18.20023242v1

42. Tang N, Li D, Wang X, Sun Z. Abnormal coagulation parameters are associated with poor prognosis in patients with novel coronavirus pneumonia. J Thromb Haemost. 2020;18:844-7.

43. Liu Q, Wang R, Qu G, Wang Y, Liu P, Zhu Y. General anatomy report of novel coronavirus pneumonia death corpse. J Forensic Med. 2020;36:19-21.

44. Iba T, Levi M, Levy JH. Sepsis-induced coagulopathy and disseminated intravascular coagulation. Semin Thromb Hemost. 2020;46:089-095.

45. Iba T, Levy J. Inflammation and thrombosis: roles of neutrophils, platelets, and endothelial cells and their interactions in thrombus formation in sepsis. J Thromb Haemost. 2018;16:231-41.

46. Lillicrap D. Disseminated intravascular coagulation in patients with 2019nCoV pneumonia. J Thromb Haemost. 2020;18:786-7.

47. Lippi G, Plebani M, Henry B. Thrombocytopenia is associated with severe coronavirus disease 2019 (COVID-19) infections: a meta-analysis. Clin Chim Acta. 2020;506:145-8.

48. Cui S, Chen S, Li X, Liu S, Wang F. Prevalence of venous thromboembolism in patients with severe novel coronavirus pneumonia. J Thromb Haemost. 2020 Apr 9. doi: 10.1111/jth.14830. Online ahead of print

49. Klok FA, Kruip MJHA, van der Meer NJM, Arbous MS, Gommers DAMPJ, Kant KM, et al. Incidence of thrombotic complications in critically ill ICU patients with COVID-19. Thromb Res. 2020 Apr 10:S0049-3848(20)30120-1. doi: 10.1016/j.thromres.2020.04.013. Online ahead of print.

50. Recalti S. Cutaneous manifestations in COVID-19: a first perspective. J Eur Acad Dermatol Venereol. 2020;34:e212-3.

51. Joob B, Wiwanitkit V. COVID-19 can present with a rash and be mistaken for dengue. J Am Acad Dermatol. 2020;82:e177.

52. Jimenez-Cauhe J, Ortega-Quijano D, Prieto-Barrios M, Moreno-Arrones OM, Fernandez-Nieto D. Reply to "COVID-19 can present with a rash and be mistaken for Dengue": petechial rash in a patient with COVID-19 infection. J Am Acad Dermatol. 2020 Apr 10;S0190-9622(20)30556-9. doi: 10.1016/j. jaad.2020.04.016. Online ahead of print.

53. Bolognia J, Schaffer J, Cerroni L. Demartología. Elservier. 4ta Edición, Barcelona, España, 2019, pp. 1425-1446.

54. Deschamps O, Ortonne N, Hüe S, Rodríguez C, Deschodt C, Hirsch G, et al. Acute exanthemas: a prospective study of 98 adult patients with an emphasis on cytokinic and metagenomic investigation. Br J Dermatol. 2019;182:355-63.

55. Manalo IF, Smith MK, Cheeley J, Jacobs R. A dermatologic manifestation of COVID-19: transient livedo reticularis. J Am Acad Dermatol. $2020 \mathrm{Apr}$ 10:S0190-9622(20)30558-2. doi: 10.1016/j.jaad.2020.04.018. Online ahead of print.

56. Mazzotta F, Troccoli T. Acute acro-ischemia in the child at the time of COVID-19. Dermatologia Pediatrica, Bari; 2020. (In press) 